\title{
Examining the influence of different types of dynamic change in a visual search task
}

\author{
Mengzhu Fu ${ }^{1} \cdot$ Logan L. Miller ${ }^{1} \cdot$ Michael D. Dodd ${ }^{1}$
}

(C) The Psychonomic Society, Inc. 2020

\begin{abstract}
It has been repeatedly demonstrated that when performing a visual search task, items can pop out of a display such that they are identified rapidly, independent of the number of distractors present. It has been less clear whether this type of pop-out is limited to static displays (e.g., images) or whether it can also occur in scenes containing movement, more akin to how we experience the real world. Recently, Jardine and Moore (Journal of Experimental Psychology: Human Perception and Performance, 42, 617-630, 2016) examined whether pop-out also occurs in displays consisting of dynamic motion - wherein items in the display rotated continuously until a critical frame that would elicit pop-out under static presentation conditions - and found that search was greatly impaired. It remains unclear, however, whether such impairment is exerted equivalently across all types of dynamic motions or if it is specific to orientation. In the present study, we replicate the original Jardine and Moore (Journal of Experimental Psychology: Human Perception and Performance, 42, 617-630, 2016) finding and extend this examination to another dimension - color change. We also explore whether search efficiency can be improved with dynamic context if aspects of the display become predictable. The results suggest that not all types of dynamic change impair search performance. Specifically, oddball color targets continue to pop out even when the items in the display are dynamic. Interestingly, adding predictable context did not aid search accuracy as expected, rather resulting in poorer performance. Taken together, the findings suggest that the influence of dynamic context on search performance is not absolute.
\end{abstract}

Keywords Attention $\cdot$ Visual search $\cdot$ Motion

\section{Introduction}

When visiting an amusement park - or any large public gathering space - it is a frequent occurrence to see small groups of individuals all wearing the same unique and brightly colored tshirt (e.g., neon yellow). The logic of this irregular clothing choice is simple: by wearing a shirt that is quite unlike anything that others in the park are wearing (outside of your immediate group), it should be easier to locate the people you are with, even in large crowds and with everyone continually moving in different directions. In other words, the intent of the bright t-shirt is to "pop out" from all the other distracting

Electronic supplementary material The online version of this article (https://doi.org/10.3758/s13414-020-02078-z) contains supplementary material, which is available to authorized users.

Mengzhu Fu

mengzhu.fu@huskers.unl.edu

1 Department of Psychology, University of Nebraska - Lincoln, Lincoln, NE, USA non-target individuals, permitting the people you are with to easily locate you and vice versa.

Though the aforementioned example is a rare occurrence for many (outside of amusement park enthusiasts), searching for a single target object among a series of distracting nontarget objects is a common task that is carried out numerous times each day. A variety of factors, however, can influence performance and lead to considerable differences in search times. Sometimes search is efficient in that rather than going through a process in which we consciously search for the target, it "pops out" automatically (e.g., a traffic light on a dark country road pops out from the night sky); other times, search is not efficient, and we have to attend to each item serially until the target is detected, which results in a more time-consuming task (e.g., trying to find a friend in the crowd at a football game when everyone in the stands is wearing the same color outfit). Treisman and Gelade (1980) suggested that at an early stage of processing we are able to perceive simple features in parallel without attention limits. As such, when the target is defined by a single salient feature (e.g., color), search is efficient regardless of the number of surrounding 
distractors, a situation commonly referred to as parallel search. On the other hand, when a target is defined by a conjunction of features, search is less efficient, and attention is required such that observers must go through each object until the target is detected. This is more commonly referred to as serial search. It has been repeatedly demonstrated that search is slower and less accurate as set size increases in serial search, whereas performance efficiency is unaffected in parallel search (e.g., Duncan \& Humphreys, 1989; Egeth \& Dagenbach, 1991; Treisman \& Gelade, 1980; Williams, Reingold, Moscovitch, \& Behrmann, 1997; Zelinsky \& Sheinberg, 1997; for a review, see Wolfe, 1998).

Pop-out in parallel search has been studied in a variety of contexts and with a number of different stimuli (for a review, see Wolfe, 1998). Most relevant to the present investigation, however, is the degree to which color has been shown to consistently pop out in displays. For example, Bravo and Nakayama (1992) had participants search for a color singleton target among a set of identical distractors and observed equivalent target detection times (on both color-categorization trials and target-present/absent trials) independent of the number of distractors in the display and consistent with a number of related findings (e.g., D’Zmura, 1991). Similarly, evidence from the guided search literature has suggested that color is a critical feature that guides attention efficiently (e.g., Duncan, 1989; D’Zmura, 1991; Green \& Anderson, 1956; Nagy \& Sanchez, 1990; Smallman \& Boynton, 1990; Treisman \& Gelade, 1980). For example, Green and Anderson (1956) had participants search for a colored two-digit number among a set of other colored two-digit numbers (distractor colors were also manipulated to be similar or dissimilar to the target color) and showed that when the target color was cued in advance, response time remained relatively constant with only a slight increase as a function of the number of other-colored distractors. This was not the case, however, when the target color was not cued in advance. Moreover, Alexander, Nahvi, and Zelinsky (2019) have recently demonstrated that while many features can be used to guide visual search, color is the most effective, with non-color features (e.g., shape) providing useful guidance only when color information is not available.

While color is a widely studied feature, pop-out has been examined as it relates to a wide variety of stimuli. Orientation is another commonly studied feature given evidence that a single oriented target can be effortlessly detected regardless of set size, provided that the distractors are all in the same orientation (Wolfe, Friedman-Hill, Stewart, \& O'Connell, 1992). More specifically, it is suggested that a tilted target line is detected more quickly among vertical lines than vice versa (Treisman, 1986; Wolfe et al., 1992). Beyond simple features, it has also been demonstrated that pop-out can be facilitated via top-down factors. For instance, Wang, Cavanagh, and Green (1994) used mirror images and had participants search for a specific target among distractors. They found that familiarity of background items aids search performance, such that faster response time was observed when an unfamiliar target (an inverted N) was embedded among familiar distractors (Ns). Extensive practice has also been shown to facilitate search performance under certain circumstances. Sireteanu and Rettenbach $(1995,2000)$ had participants perform serial search tasks repeatedly over an extended period and found that response time was significantly reduced after training, with a pattern of results more akin to parallel search.

Though the aforementioned studies provide considerable evidence that stimuli in our visual environment can "pop out" in a variety of ways, it is noteworthy that these investigations present target stimuli as static images that do not change at any point during each trial. In the real world, however, it is rarely the case that all of the stimuli in our visual field are static. Returning to our amusement park example, it is not the case that the people and objects in your visual field remain motionless as you search for the people you are with. Both targets (your group of friends) and distractors (other people and other irrelevant stimuli in the park) are constantly changing position, requiring us to search for the desired target information in a dynamic context (e.g., the uniquely colored shirt must continue to stand out even as the surrounding context rapidly changes). This leads to the question of whether stimuli similarly pop out in dynamic environments, more akin to what we constantly experience in everyday life. Jardine and Moore (2016) recently investigated whether these effects could also be observed in dynamic displays given evidence that scene dynamics influence perception (Hollingworth \& Henderson, 2000). These researchers created systematically changing scenes in which a standard static pop-out display (a single item has a unique orientation relative to all other distractors, a situation that commonly elicits pop-out in static search) was modified to incorporate motion by having elements rotate in place. Video 1 in the Supplementary Online Materials presents an example display used in the current study that resembles the original stimuli of Jardine and Moore (2016). At the critical frame (identical to a static pop-out display that should yield a highly efficient search) in which each distractor is either a vertical or a horizontal bar with the target being an oblique bar - participants were asked to report the direction of the target (tilted to the left or right). Multiple set sizes (16 and 32), frames of animation/rotation (1, 3,5 , and 11) and frame durations (speed at which the bars rotate; 100-800 ms) were used (Jardine \& Moore, 2016). Though the presentation duration of a standard static popout display and the critical frame of the dynamic display were identical, highly efficient pop-out was only observed in static displays, with accuracy being near chance with the dynamic displays. Though performance improved with increasingly long frame durations (slower rotation), it never matched the level of that observed in static displays. 
Jardine and Moore (2016) provide convincing evidence that dynamic displays impair pop out; however, it is important to note that each of their experiments required an orientation judgment, making it unclear whether all dynamic displays prevent pop out, or whether this deficit is specific to orientation. Pop-out has been investigated with a number of different visual displays and the generality of the effect is unclear if only examined within a single paradigm.

The purpose of the present investigation was to extend the study of pop-out in dynamic displays to judgments beyond line orientation. Given that pop-out has been examined in a number of different contexts, it was unclear which factors were relevant to the real-world and would be more/less likely to pop out. As such, we initially conducted a pilot investigation in which participants completed blocks of trials that were adapted from the general paradigm of Jardine and Moore (2016) (see Appendix A). Extending from the original study, we included different dynamic target and distractor stimuli in each block (e.g., orientation change, color change, emotion change). Given the range of factors that might influence pop-out, the pilot was run with the purpose of determining whether there were meaningful variations in search performance that would afford us the opportunity to preregister the experiment reported in the present paper. Consistent with the findings of Jardine and Moore (2016), the pilot experiment observed impaired search accuracy in dynamic contexts involving orientation change. We also found preliminary evidence suggesting that introducing top-down expectations may aid search performance. More interestingly, superior search performance was observed in color-change conditions where objects were changing shades arbitrarily. This suggests that color pop-out may be somewhat resistant to the detrimental effect of dynamic context that is observed with line orientations, consistent with studies demonstrating that color is a critical guiding feature (e.g., Alexander et al., 2019). However, it is important to note that the manner in which the color of each item changed in the pilot study was random rather than systematic. It is possible, therefore, that the display items were not being viewed as single objects that were continually changing at fixed locations inasmuch as different objects that were being presented rapidly at each location.

\section{Current investigation}

While our pilot data were consistent with the notion that color continues to pop out - even in dynamic situations - the investigation was limited in scope and included a number of additional exploratory conditions that are not relevant to the current investigation. As such, we sought to preregister the current study using the pilot data as the basis for our predictions, analyses, and adapted method. To that end, the current study was registered to the Open Science Framework (osf.io/shqxr) to further investigate the influence of dynamic motion on visual search performance. There were three critical goals. First, we sought to replicate Jardine and Moore (2016) in examining the impact of dynamic motion on pop out of orientation. In the orientation condition, bars rotate clockwise or counterclockwise in place, and participants are asked to detect an oblique bar during a critical frame. Second, we extend the examination of dynamic context on pop-out to color given evidence in our pilot data that color pop-out may be immune to the impairment observed for orientation. Importantly, color change in the present experiment was manipulated in a sequential rather than a random fashion to ensure that display items were being perceived as single objects that were changing gradually in appearance. We expected pop-out to be equivalent in static and dynamic displays when color comprised the target, but impaired when a tilted line comprised the target. Finally, we sought to more fully investigate whether the perception of pop-out in dynamic displays could be improved via a predictable context (top-down expectancy), affording a determination of when the target frame was about to occur. It has been previously demonstrated that participants can prioritize novel items in a display when they have previously been exposed to distractors/distractor locations (Watson \& Humphreys, 1998). Therefore, knowing when the critical frame is set to occur may allow participants to better anticipate which items are likely to be targets/distractors.

\section{Method}

This project was registered on the Open Science Framework (osf.io/shqxr). All details regarding sample size, experimental conditions, and analyses were determined in advance of data collection.

\section{Participants}

Forty-two undergraduate students from the University of Nebraska - Lincoln participated in the experiment in exchange for course credit ( 30 were targeted for data collection but participants were replaced if they did not finish all of the conditions or failed to follow instructions as described below). All participants had normal or corrected-to-normal vision and were naïve to the purpose of the experiment, which took place in a single 60-min session.

\section{Apparatus}

All stimuli were programmed using Psychtoolbox in MATLAB (R2017b). The experiment was conducted on Windows 10 64-bit computers (Intel Xeon E5603 CPU, with 1.6-Hz quad-core and an NVIDIA Quadro 600 1-GB graphics card). The monitor was 23 -in. with a resolution of $1,920 \times$ 
1,080 pixels. Participants were seated in front of the computer screen at a viewing distance of approximately $60 \mathrm{~cm}$.

\section{Design}

The study was conducted using a 3 (cue type: orientation, color, or predictability) $\times 2$ (display type: static vs. dynamic) $\times 2$ (set size: 16 vs. 32$) \times 2($ stimulus onset asynchrony $(\mathrm{SOA})$ : $400 \mathrm{~ms}$ vs. $800 \mathrm{~ms}$ ) repeated-measures design. The experiment consisted of four blocks, three of which used dynamic displays, each containing one cue type; the remaining condition used static displays, which contained all three cue types. Participants first completed the three dynamic conditions in a randomly assigned order. The static condition was always the last to be completed.

\section{Materials and procedure}

After giving consent to participate in the study, participants were instructed to sit in front of a computer screen and were provided task instructions. Figure 1 shows example trials (up until the critical frame) for each of the dynamic conditions (see Videos 1, 2, and 3 in the Supplementary Online Materials for animated examples). Trials began with the search display, which could consist of either colored circles or tilted lines. In the gradual color change condition, colored circles systematically changed shades; in the orientation condition, bars rotated clockwise or counterclockwise; and in the predictability condition, the displays were identical to the orientation condition except that an additional colored border appeared around each frame that denoted when the critical frame would occur. The critical frame was the middle frame in the sequence in all three conditions. The main measurement of interest was accuracy (\%), though response time was also recorded and analyzed.

\section{Orientation condition}

The orientation condition used the same target/distractor type and configuration as Jardine and Moore (2016), which comprised a series of rotating bars. Each trial consisted of a sequence of seven frames, with 16 or 32 items presented on randomly chosen cells of an invisible $6 \times 6$ grid. Each bar subtended approximately $1^{\circ}$ of visual angle. The orientation of the bars changed $12.5^{\circ}$ clockwise or counterclockwise at every frame change (half of the bars rotated clockwise and the other half of bars rotated counterclockwise on each trial), with a duration of $800 \mathrm{~ms}$ or $400 \mathrm{~ms}$. These two frame durations were chosen because they were used in the original Jardine and Moore (2016) study, with the 800-ms frame duration eliciting accuracy over $80 \%$ (relative to greater impairment with shorter frame durations), making it ideal for comparison purposes to determine whether other dynamic contexts would lead to similar performance or facilitated/impaired performance. At the critical frame (fourth frame in the sequence), the display contained an oblique bar (target) among a crowd of horizontal and/or vertical bars (distractors), with a white border appearing around the stimuli. Participants were asked to

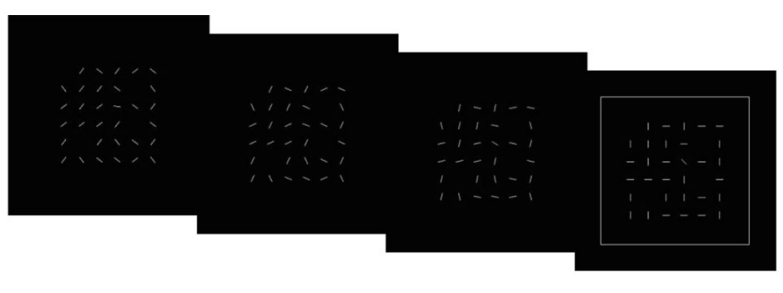

Orientation Condition

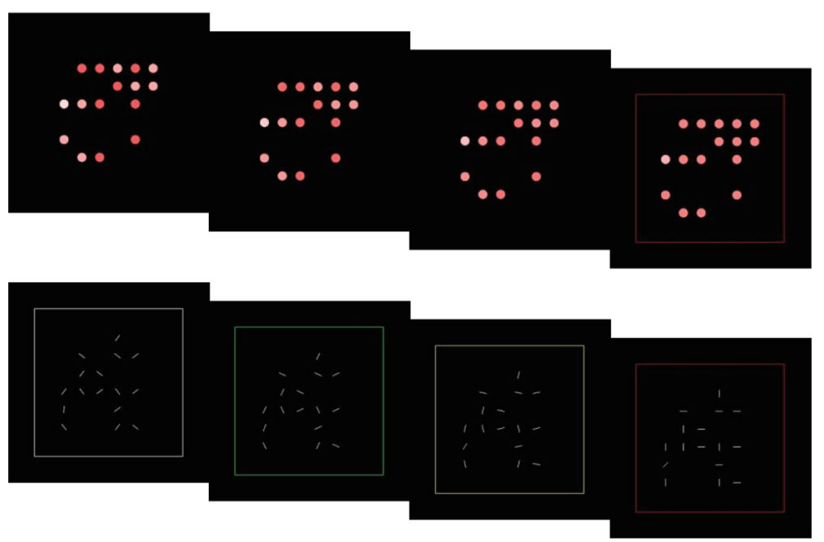

Color Condition

Predictability Condition

Fig. 1 Example trial sequence. Only the first four frames are displayed with the colored border in frame 4 (white in the orientation condition, red in the color and predictability conditions) indicating the critical "frame" for each condition 
identify which side of the screen the target was on. They were allowed to make a response as soon as the onset of the critical frame, and up to $2 \mathrm{~s}$ after the sequence ended. Participants pressed $\mathrm{F} / \mathrm{J}$ key in correspondence with the left/right side of the screen. Video 1 in the Supplementary Online Materials presents an example trial of the orientation condition. Participants first completed a practice block (five trials), and then completed four experimental blocks. In each block, one of the four trial types (two set sizes $\times$ two SOAs) was displayed. Each block contained 40 trials, making up a total of 160 trials.

\section{Color condition}

In the previous pilot investigation, we utilized a random-color change in which the hue of each color could turn lighter or darker in an unpredictable fashion. This, however, was inconsistent with the manner in which stimuli changed in the orientation conditions (i.e., stepwise rotation and change magnitude is constant). One concern regarding the pilot study was that the high accuracy was attributable to participants perceiving the presentation of multiple different objects at a single location rather than as a single item at a fixed location that is changing color dynamically. To make all conditions as similar as possible, we altered the manner in which color changed in the present experiment to a more gradual and consistent change. The color change condition in the current study utilized red-shaded circles. Each trial consisted of a sequence of seven frames, with 16 or 32 items presented on randomly chosen cells of an invisible $6 \times 6$ grid (see Video 2 in the Supplementary Online Materials for an example trial). The diameter of each red circle was 50 pixels (visual angle $=$ $1.26^{\circ}$ ), and with each frame change, the saturation percentage of each colored circle moved up or down by $5 \%$ (range is between $15 \%$ and $65 \%$ ), making the circles look darker or lighter (the saturation decreased for half of the circles, while it increased for the other half of the circles on each trial). At the critical frame, a red border appeared around the stimuli, and participants were asked to detect a circle that had a unique shade (target) amidst a series of distractors that were all the same shade. Participants were asked to identify which side of the screen the target was on. Responses could be made as soon as the onset of the critical frame, and up to $2 \mathrm{~s}$ after the sequence was over. Participants first completed a practice block, and then completed four experimental blocks, each of which contained 40 trials, making up 160 trials in total. In each block, one of the four trial types (two set sizes $\times$ two SOAs) was displayed.

\section{Predictability condition}

The stimuli and setup in the predictability condition were identical to those in the orientation condition with the exception that a colored square border would appear with every frame change. The order of border-color changes was constant and predictable, with participants being informed of the following order: white, green, yellow, red, yellow, green, and white. The onset of the red frame indicated the presence of the critical frame, meaning that participants could anticipate when they would be responding to the display (see Video 3 in the Supplementary Online Materials for an example trial). We sought to determine whether explicitly providing participants foreknowledge of when to expect the onset of the critical frame may improve search performance. Studies on preview search (Braithwaite \& Humphreys, 2003; Olivers, Humphreys, \& Braithwaite, 2006; Watson \& Humphreys, 1998) have repeatedly demonstrated that target detection can be facilitated if participants are able to "preview" a subset of distractors prior to the critical search display. The logic here is similar in that by providing participants with the foreknowledge about when the critical frame occurs, along with the constant within-trial speed of rotation, they may begin to get a sense of which items are more likely to be targets/distractors. Similar to the orientation condition and the gradual color change condition, participants first completed a practice block, followed by four experimental blocks. Each block contained 40 trials, making up 160 trials in total. In each block, one of the four trial types (two set sizes $\times$ two SOAs) was displayed.

\section{Static control condition}

The static control condition contained three types of stimuli, which were identical to the ones used in the three dynamic conditions, with the following exceptions: in the static condition, instead of presenting the whole sequence of stimuli, only the critical frame was presented. Note that the only difference between the static orientation condition and static predictability condition was the color of the border around the stimuli (white in the orientation condition and red in the predictability condition), and this was not expected to impact performance in any way. Participants searched for a target among distractors and were given up to $2 \mathrm{~s}$ to make a response. No practice block was given in this condition and pop-out was expected to be observed with all static displays. Participants completed 12 experimental blocks, each of which contained one of the 12 trial types (three cue types $\times$ two set sizes $\times$ two SOAs). Each block contained 20 trials, making up a total of 240 trials.

\section{Results}

Twelve participants were excluded from the sample due to incomplete data or failure to follow the instructions described above. Practice trials were also not analyzed. Values below 
$150 \mathrm{~ms}$ or larger than 2.5 standard deviations above the mean response time were considered outliers and were removed from the dataset. In total, $6.9 \%$ of trials were excluded from analysis.

\section{Search accuracy analysis}

A four-way repeated-measures ANOVA was then run to compare the mean accuracy (percent correct) across conditions. Descriptive information about mean percentage of correct responses is reported in Table 1.

Overall, percentage of correct responses differed significantly between dynamic conditions and static conditions, $F(1,29)=16.19, p<.001, \eta_{p}{ }^{2}=.36$. Participants were more accurate at detecting targets in the static displays $(M=92.0 \%$, $S D=4.8)$ than in the dynamic ones $(M=88.9 \%, S D=5.8)$, as would be expected given the results of Jardine and Moore (2016). There were also main effects of SOA, $F(1,29)=$ $139.80, p<.001, \eta_{p}{ }^{2}=.83$, with participants being more accurate at longer SOAs $(M=94.3 \%, S D=4.2)$ than at shorter SOAs $(M=86.7 \%, S D=6.0)$. Interestingly, there were main effects of set size, $F(1,29)=9.58, p=.004, \eta_{p}{ }^{2}=.25$, with participants being slightly better at detecting targets in 32-item displays $(M=91.3 \%, S D=4.6)$ than in 16-item displays $(M=$ $89.7 \%, S D=5.4)$. Critically, variability in search performance was observed across different cue types, $F(2,58)=44.10, p<$ $.001, \eta_{p}{ }^{2}=.60$. Pairwise-comparisons revealed that the highest accuracy was observed in the color condition ( $M$ $=94.5 \%, S D=4.4)$, followed by the orientation condition $(M$ $=89.7 \%, S D=5.8)$, and lastly the predictability condition $(M$ $=87.3 \%, S D=5.9$ ).

There was an interaction between display and cue types (see Fig. 2), $F(2,58)=45.79, p<.001, \eta_{p}{ }^{2}=.61$. Bonferroni-corrected pairwise comparisons revealed that while search accuracy remained relatively high in the color conditions, performance was slightly better in the dynamic color displays $(M=96.5 \%, S D=4.4)$ relative to the static color displays $(M=92.4 \%, S D=5.1), t(29)=$ $6.33, p<.001$. In addition, overall performance was significantly better in the static versus dynamic displays as it relates to the orientation $(M=91.5 \%, S D=5.8$ and $M=$ $87.9 \%, S D=7.7, t(29)=2.85, p=.008)$ and predictability conditions $(M=92.2 \%, S D=5.1$ and $M=82.4 \%, S D=$ $8.5, t(29)=7.20, p<.001)$. The decrement of accuracy in the dynamic predictability condition suggests that contrary to the prediction that providing foreknowledge about when to expect the onset of the critical frame would aid search performance, search accuracy was in fact negatively impacted by this additional information.

An interaction between cue type and SOA was also observed (see Fig. 3), $F(2,58)=12.80, p<.001, \eta_{p}{ }^{2}=.31$. Follow-up pairwise comparisons using Bonferroni correction showed that search accuracy was always higher in the color condition compared to the other two conditions, regardless of frame duration ( $p<.001$ for all related comparisons). Pairwise comparison further revealed that while there was no significant difference between the orientation condition $(M=93.4 \%$, $S D=5.0)$ and predictability condition $(M=92.6 \%, S D=5.8)$ when frame duration was $800 \mathrm{~ms}(p=.56)$, performance was significantly better in the former $(M=86.0 \%, S D=7.1)$ than the latter $(M=82.0 \%, S D=7.6)$ when frame duration was 400 ms, $t(29)=3.90, p=.002$.

Further, there was an interaction between set size and display, $F(1,29)=7.27, p=.012, \eta_{p}{ }^{2}=.20$. Follow-up pairwise comparisons revealed that overall accuracy remained consistently lower in dynamic conditions (relative to static conditions) regardless of the display set size ( $M=88.9 \%$ and $89.0 \%, S D=6.2$ and $6.1, p=.87$ ). In the static conditions on the other hand, performance was slightly (though significant, $t(29)=4.12, p<.001)$ better with a larger set size $(M=$ $93.5 \%, S D=4.0)$ than with the smaller set size $(M=90.5 \%$, $S D=6.1)$.

Table 1. Descriptive statistics: Mean accuracy (\%) as a function of display type (dynamic vs. static), cue type (color, orientation, predictability), set size (16 vs. 32), and frame duration (400 vs. $800 \mathrm{~ms}$ ). Standard deviations appear in parentheses beside each mean

\begin{tabular}{|c|c|c|c|c|}
\hline \multirow{5}{*}{$\begin{array}{l}16 \text { items } \\
32 \text { items }\end{array}$} & \multicolumn{4}{|c|}{ Color } \\
\hline & \multicolumn{2}{|c|}{ Dynamic } & \multicolumn{2}{|c|}{ Static } \\
\hline & $400 \mathrm{~ms}$ & $800 \mathrm{~ms}$ & $400 \mathrm{~ms}$ & $800 \mathrm{~ms}$ \\
\hline & $95.8(6.2)$ & $97.7(3.6)$ & $87.3(9.7)$ & $94.4(6.2)$ \\
\hline & $94.6(10.2)$ & $98.2(3.6)$ & $91.0(8.4)$ & $96.8(3.6)$ \\
\hline \multirow{5}{*}{$\begin{array}{l}16 \text { items } \\
32 \text { items }\end{array}$} & \multicolumn{4}{|c|}{ Orientation } \\
\hline & \multicolumn{2}{|c|}{ Dynamic } & \multicolumn{2}{|c|}{ Static } \\
\hline & $400 \mathrm{~ms}$ & $800 \mathrm{~ms}$ & $400 \mathrm{~ms}$ & $800 \mathrm{~ms}$ \\
\hline & $81.5(13.4)$ & $92.7(5.5)$ & $88.4(10.5)$ & $90.8(8.2)$ \\
\hline & $82.7(9.4)$ & $94.5(8.1)$ & $91.2(7.8)$ & $95.8(4.6)$ \\
\hline & \multicolumn{4}{|c|}{ Predictability } \\
\hline & \multicolumn{2}{|c|}{ Dynamic } & \multicolumn{2}{|c|}{ Static } \\
\hline & $400 \mathrm{~ms}$ & $800 \mathrm{~ms}$ & $400 \mathrm{~ms}$ & $800 \mathrm{~ms}$ \\
\hline 16 items & $76.5(11.9)$ & $89.1(13.1)$ & $88.1(9.1)$ & $94.3(7.6)$ \\
\hline 32 items & $72.5(14.3)$ & $91.4(8.6)$ & $90.9(8.0)$ & $95.5(5.4)$ \\
\hline
\end{tabular}




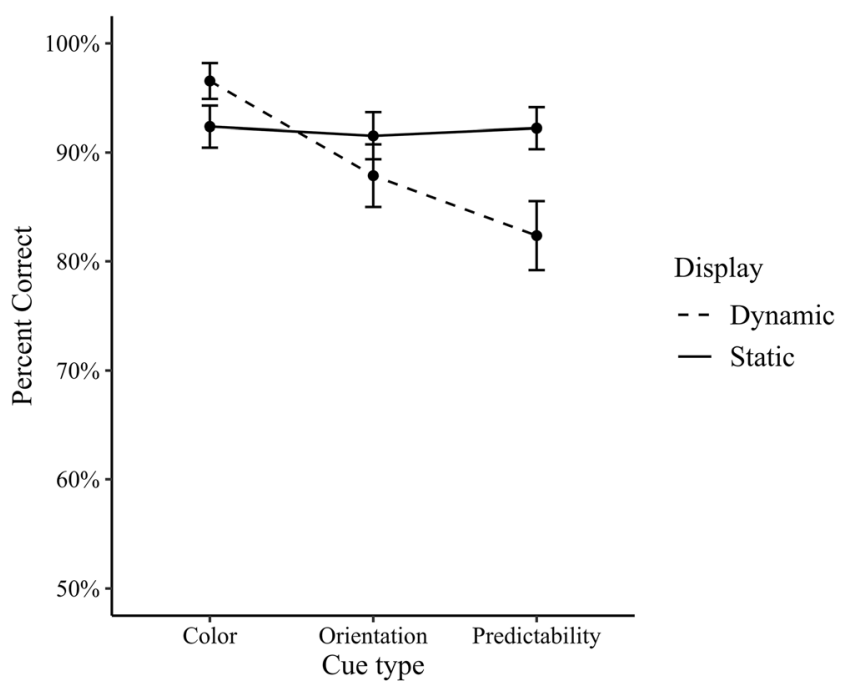

Fig. 2 Mean search accuracy as a function of cue type and display type. The error bars represent $95 \%$ confidence intervals. Highest accuracy was observed in the color condition, followed by the orientation condition and the predictability condition. Overall, search performance was better in static displays relative to dynamic displays, with the exception of the color condition

An interaction between display and SOA was also observed, $F(1,29)=26.77, p<.001, \eta_{p}{ }^{2}=.48$. Pairwise comparisons revealed that while performance was not significantly different between static $(M=94.6, S D=3.9)$ and dynamic displays $(M=93.9, S D=5.3)$ when SOA was $800 \mathrm{~ms}(p=$ .39), performance was significantly better in the static displays $(M=89.5 \%, S D=6.2)$ compared to the dynamic displays $(M$ $=83.9 \%, S D=7.0)$ with shorter frame duration, $t(29)=5.33, p$ $<.001$.

Lastly, there was a three-way interaction between cue type, SOA, and display (Fig. 4 and Fig. 5), $F(2,58)=15.28, p<.001$, $\eta_{p}{ }^{2}=.35$. Critically, while performance remained relatively high in the color condition, accuracy was better at the 800 -ms frame duration $(M=97.9 \%, S D=3.5)$ than at $400-\mathrm{ms}(M=$ $95.2 \%, S D=6.5)$ in both dynamic $(t(29)=2.68, p=.012)$ and static color displays $(M=95.6 \%$ and $89.2 \%, S D=4.1$ and 7.3 , $t(29)=5.94, p<.001)$. In the orientation condition, dynamic displays impaired performance with the 400 -ms presentation $(M=82.1 \%, S D=10.2)$, whereas search accuracy was significantly higher at the longer frame duration $(M=93.6 \%, S D=$ $6.0, t(29)=9.37, p<.001)$. Similar results were also observed in the static orientation displays $(M=93.3 \%$ and $89.8 \%, S D=$ 5.3 and 7.8), though to a lesser extent, $t(29)=2.81, p=.009$. This is consistent with Jardine and Moore (2016), who observed enhanced performance at longer presentation times. While a similar pattern of results was also observed in both the static $(M=94.9 \%$ and $89.5 \%, S D=5.0$ and $6.8, t(29)=$ $4.78, p<.001)$ and dynamic predictability conditions $(M=$ $90.3 \%$ and $74.5 \%, S D=8.7$ and $11.2, t(29)=8.09, p<.001)$, accuracy in these conditions was lower across the board, with a greater decrement at the shorter frame duration.

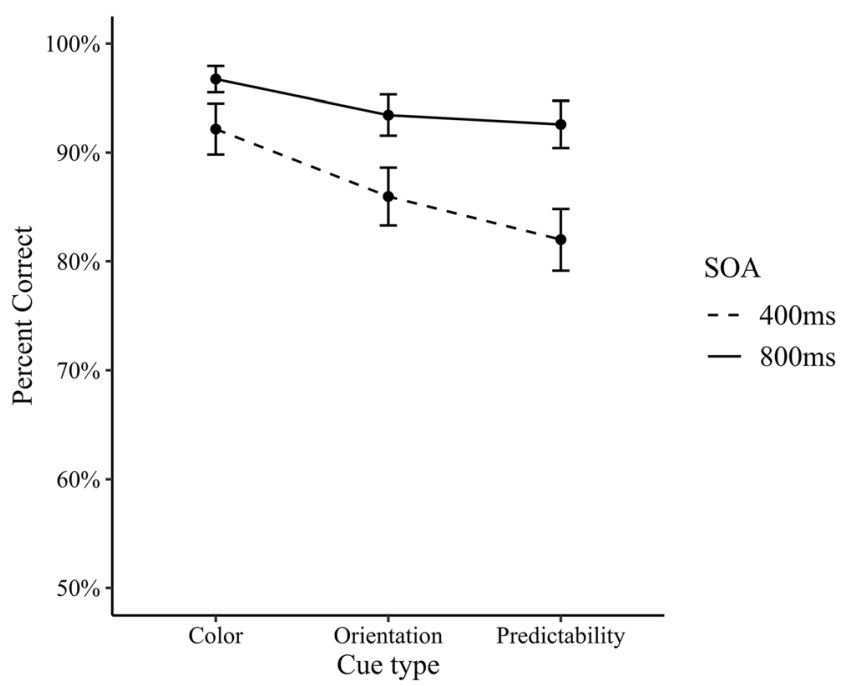

Fig. 3 Mean search accuracy as a function of cue type and frame duration. The error bars represent $95 \%$ confidence intervals. Highest accuracy was observed in the color condition, followed by the orientation condition and the predictability condition. Overall, higher accuracy was observed with longer frame duration. However, search performance was not significantly different between the orientation condition and predictability condition when frame duration was 800 $\mathrm{ms}$, while better performance was observed in the former when frame duration was $400 \mathrm{~ms}$

\section{Response-time analysis}

Identical analyses were also carried out on the responsetime data despite accuracy being the critical measure (descriptive statistics are shown in Table 2 and ANOVA results in Table 3), and the results further supported findings observed for performance accuracy. Most interestingly, there was an interaction between display and cue type,

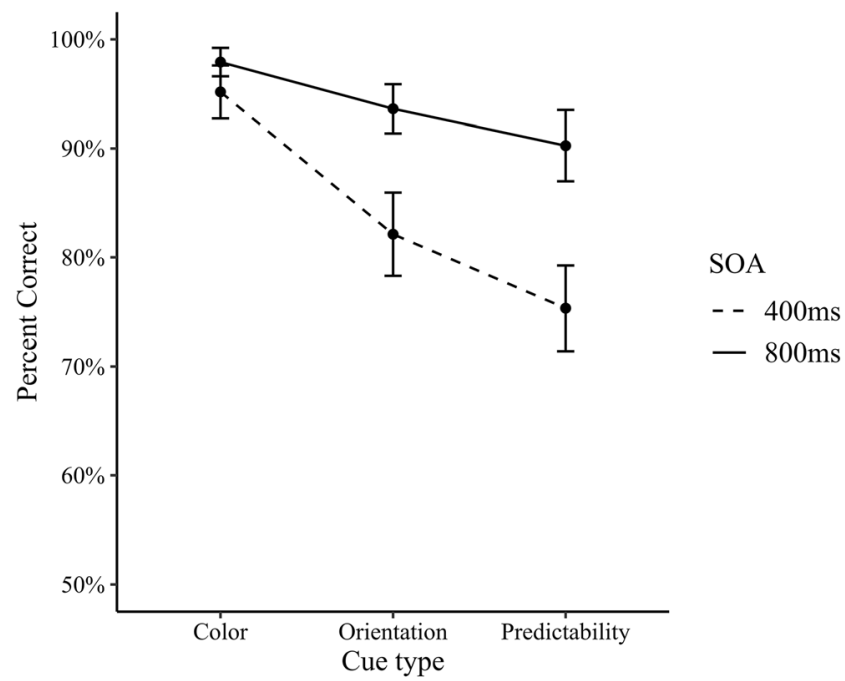

Fig. 4 Mean search accuracy as a function of cue type and frame duration with dynamic displays. The error bars represent $95 \%$ confidence intervals. Highest accuracy was observed in the color condition, followed by the orientation condition and the predictability condition. Search performance was significantly higher at $800-\mathrm{ms}$ frame duration than at 400-ms frame duration with all three cue types 


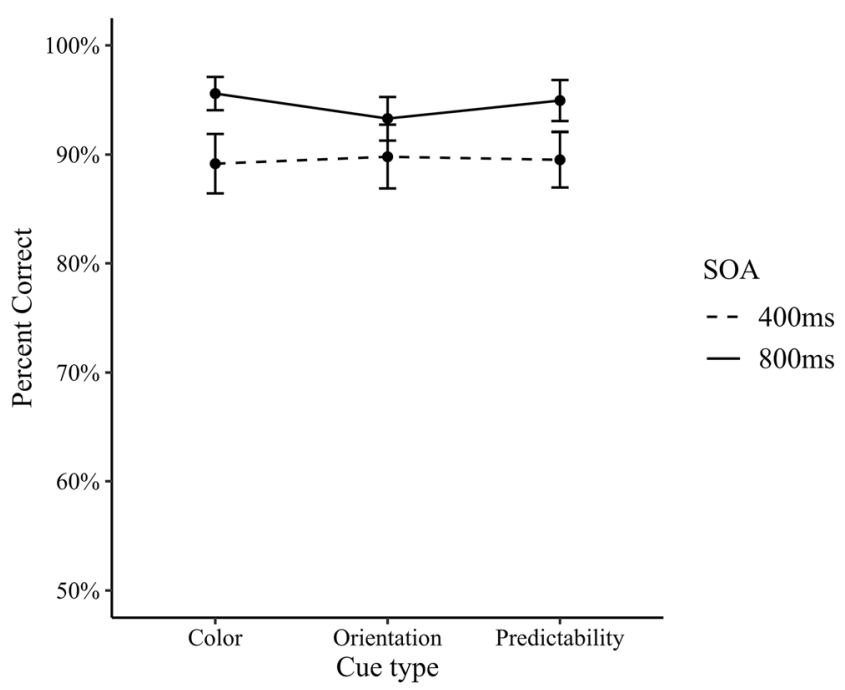

Fig. 5 Mean search accuracy as a function of cue type and frame duration with static displays. The error bars represent $95 \%$ confidence intervals. Overall, higher search accuracy was observed with longer frame duration with all three cue types

$F(2,58)=43.16, p<.001, \eta_{p}^{2}=.60$. Overall, faster responses were observed in static displays $(M=551.4, S D=$ 61.7) than in dynamic displays $(M=773.4, S D=128.4)$. Furthermore, the color condition yielded the fastest response $(M=518.9, S D=78.4)$, followed by the orientation condition $(M=713.2, S D=87.9)$, and then the predictability condition $(M=755.1, S D=144.3)$. More importantly, follow-up pairwise comparison revealed no difference between the static and dynamic color conditions $(p=.24)$ as it relates to response time. However, participants in general took significantly longer time to detect the target in dynamic displays for both the orientation $(M=854.1$ and $572.4, S D=149.4$ and $73.7, t(29)=9.84, p<.001)$ and predictability conditions $(M=930.9$ and $579.4, S D=225.7$ and 79.7, $t(29)=10.88, p<.001)$. The results thus suggest that color consistently pops out in both static and dynamic
Table 3. Response time: ANOVA statistics for response-time data

\begin{tabular}{llllll}
\hline Effect & DF $_{\text {effect }}$ & $\mathrm{DF}_{\text {error }}$ & $\mathrm{F}$ & $\mathrm{p}$ & $\eta_{p}{ }^{2}$ \\
\hline Display type & 1 & 29 & 113.39 & $<.001$ & .80 \\
Cue type & 2 & 58 & 67.98 & $<.001$ & .70 \\
Display $\times$ Cue & 2 & 58 & 43.16 & $<.001$ & .60 \\
Set size $\times$ SOA & 1 & 29 & 9.17 & .005 & .24 \\
Cue $\times$ Set size $\times$ SOA & 2 & 58 & 4.04 & .023 & .12 \\
\hline
\end{tabular}

$D F$ degrees of freedom, $S O A$ stimulus onset asynchrony

displays, as reflected in participants' equivalent response times. On the other hand, the significantly slower response time in both dynamic orientation condition and predictability condition suggests that, consistent with Jardine and Moore (2016), dynamic motion impairs search performance in orientation displays, which results in serial search.

\section{Discussion}

In the present study, we sought to determine whether the impairment in pop-out in dynamic displays that was first reported by Jardine and Moore (2016) extends to other display types or whether this was primarily restricted to displays where orientation was the critical target feature. Moreover, we examined whether search performance in dynamic displays could be facilitated if the critical frame was made predictable. While we successfully replicated Jardine and Moore (2016), observing poorer search accuracy under dynamic conditions for targets defined by orientation, consistent pop-out was observed when color was the defining target feature. This finding appears consistent with the recent findings of Alexander et al. (2019), who provided evidence that color is a critical factor in guiding

Table 2. Descriptive statistics: Mean response time (ms) as a function of display type (dynamic vs. static), cue type (color, orientation, predictability), set size (16 vs. 32), and frame duration (400 vs. $800 \mathrm{~ms}$ ). Standard deviations appear in parentheses beside each mean

\begin{tabular}{|c|c|c|c|c|}
\hline \multirow{5}{*}{$\begin{array}{l}16 \text { items } \\
32 \text { items }\end{array}$} & \multicolumn{4}{|c|}{ Color } \\
\hline & \multicolumn{2}{|c|}{ Dynamic } & \multicolumn{2}{|c|}{ Static } \\
\hline & $400 \mathrm{~ms}$ & $800 \mathrm{~ms}$ & $400 \mathrm{~ms}$ & $800 \mathrm{~ms}$ \\
\hline & $500.6(195.7)$ & $538.3(122.4)$ & $479.2(73.3)$ & $523.7(68.7)$ \\
\hline & $561.3(240.1)$ & $541.3(164.5)$ & $492.4(70.7)$ & $514.6(79.6)$ \\
\hline & \multicolumn{4}{|c|}{ Orientation } \\
\hline & \multicolumn{2}{|c|}{ Dynamic } & \multicolumn{2}{|c|}{ Static } \\
\hline & $400 \mathrm{~ms}$ & $800 \mathrm{~ms}$ & $400 \mathrm{~ms}$ & $800 \mathrm{~ms}$ \\
\hline 16 items & $854.6(230.7)$ & $861.8(141.1)$ & $562.5(106.7)$ & $586.3(98.2)$ \\
\hline \multirow[t]{4}{*}{32 items } & $840.7(190.2)$ & $859.2(142.1)$ & $553.1(91.3)$ & $587.7(91.0)$ \\
\hline & \multicolumn{4}{|c|}{ Predictability } \\
\hline & \multicolumn{2}{|c|}{ Dynamic } & \multicolumn{2}{|c|}{ Static } \\
\hline & $400 \mathrm{~ms}$ & $800 \mathrm{~ms}$ & $400 \mathrm{~ms}$ & $800 \mathrm{~ms}$ \\
\hline 16 items & $867.7(245.8)$ & $978.2(248.0)$ & $565.6(90.0)$ & $612.6(109.5)$ \\
\hline 32 items & $968.4(366.9)$ & $909.2(202.8)$ & $562.7(74.7)$ & $576.8(104.4)$ \\
\hline
\end{tabular}


search. The present results suggest that the strength of this feature is somewhat resistant to the otherwise detrimental effects of dynamic motion on pop-out, such that color changes may elicit pop-out in a manner that other types of features do not. This is also consistent with evidence demonstrating that color processing begins early and provides effective information for object perception and visual search. For instance, Hayakawa, Kawai, and Masataka (2011) showed children both color and grayscale photos of snakes, and found that children responded to the photo of snake faster when it was in color compared to when it was gray-scale. Electrophysiological studies (e.g., Proverbio, Burco, Del Zotto, \& Zani, 2004) have also shown that our brain processes color more quickly than other features (e.g., shape, orientation).

To account for the general search impairment in dynamic displays, Jardine and Moore (2016) proposed an object-based updating hypothesis, which suggests that a moving object in a dynamic scene (e.g., driving toward a stop sign) is encoded as a sequence of snapshot-like pictures. The mental representation of the object is thus updating continuously, with a newer representation being integrated into (or overwriting) the previous one. Attempts to retrieve an old representation of the object from the sequence may fail due to the overwriting process, which leads to the poorer accuracy and longer response time. Specifically, bars in the orientation condition rotate in place and are perceived as single objects changing states continuously. The pop-out frame, however, is a single frame of the changing scene, and failure to access that instantaneous state is due to the fact that it has already been overwritten by other representations.

Based on this hypothesis, one possibility for the continued color pop-out may be that color perception is qualitatively different from orientation perception. While perceptual continuity is maintained with bar rotation, color changes may be encoded in a more categorical fashion (e.g., pink vs. red), which disrupts the perception of single unified objects changing states continuously. Research in color segregation has shown that when the color of the target item is revealed to participants prior to the trial, search time remains constant regardless of the number or the color(s) of distractors in the display (e.g., Carter, 1982; Green \& Anderson, 1956; Smallman \& Boynton, 1990). While only within-category color change was utilized in the current study, future studies may consider examining color pop-out across the color spectrum. However, given the evidence provided in the color segregation literature, we would expect to observe similar results even when the color change is systematic through the color space (i.e., across hues) instead of within a single hue.

While the predictability condition in the current study was based on the premise that providing additional information regarding when the critical frame was about to occur may improve performance, this was not the case. The addition of a predictable frame led to both poorer accuracy and slower response times. One plausible explanation would be that introducing a top-down expectation in fact consumes extra cognitive capacity, which in turn impairs search performance. It may simply be that attending to both the frame changes and search displays sufficiently taxed attentional capacity and did not afford the opportunity to determine which items may be targets/distractors in advance.

With that said, another possible alternative is that the reduced search accuracy in the predictability condition may be due to the use of cognitive strategy. Studies examining the relationship between cognitive control and visual search performance have suggested that using an active search strategy (more executive control) can sometimes result in worse performance (e.g., Smilek, Enns, Eastwood, \& Merikle, 2006). Smilek et al. (2006) had participants complete both an easy and a difficult search task and found that those who were asked to passively let their "gut feeling" lead searches exhibited more efficient performance when the task was difficult relative to those who were asked to "actively" search for the target in the same experimental display. This was confirmed in a second experiment in which the researchers limited participants' executive control ability in the search task by having them complete a memory test simultaneously (i.e., increment of cognitive load). Those who were forced to perform both tasks simultaneously exhibited more efficient performance when the search task was difficult compared to those who performed the search task without a secondary requirement. The researchers therefore proposed that reduced executive control (e.g., passive search) may benefit search performance. Eye-movement studies further support this idea by showing that the use of active search strategy results in more saccades compared to those who are encouraged to search for target passively (Watson, Brennan, Kingstone, \& Enns, 2010). Participants doing passive search tended to wait longer before making their first eye movement and generally made fewer fixations. Given that fixation duration is correlated with the degree to which information processing is facilitated (e.g., Boot, Becic, \& Kramer, 2009; Boot, Kramer, Becic, Wiegmann, \& Kubose, 2006), an increase in the number of fixations may actually decrease the quality of information processing. Given the evidence presented above, it may be that impaired search performance in the predictability condition occurred because participants were motivated to actively search for the target because of the provided "hint" about when a critical frame was about to occur. Due to the task difficulty, this encouragement of active search may lead to worse performance efficiency. Taken together, the results of the present study suggest that pop-out can still occur in dynamic environments, but that it may be limited to the simple feature of color (i.e., not all dynamic contexts are equal). 
Author Note This research was supported by a grant from EPSCoR Research Infrastructure award from the National Science Foundation. Award Number 1632849RII Track-2 FEC: Neural networks underlying the integration of knowledge and perception.

Open Practices Statement The current study was preregistered on the Open Science Framework (osf.io/shqxr). The data are available on request from the corresponding author.

\section{Appendix A}

The pilot experiment adapted the general paradigm used in the original Jardine and Moore (2016) study. Methodological details were consistent with methods described in the larger experiment reported in the main text. In the pilot experiment, trials were blocked based on stimulus type and participants were asked to respond to a unique target among identical distractors at the critical frame (denoted by a border around the display). An $800-\mathrm{ms}$ frame duration was utilized as it elicited accuracy over $80 \%$ in the original Jardine and Moore (2016) study, which would allow us to compare performance across display types to determine whether all dynamic contexts lead to similar facilitated/impaired performance. Participants viewed a series of frames of each dynamic display with the critical search display being noted by a colored border around the search display. In the table below, we report the descriptive statistics for performance on three separate stimulus conditions: an orientation condition that replicated Jardine and Moore (2016), a color condition in which the rotating bars were replaced by a red color patch that continuously changed shade in a random manner, and a predictability condition in which the critical frame could be anticipated (but which otherwise perfectly mirrored the orientation condition). The critical frame in the predictability condition is made predictable via a series of sequential colored square frames indicating when the critical frame would occur. Two set sizes ( 8 vs. 16) were utilized in the predictability condition.

As would be expected, the orientation condition replicated the pattern of results observed by Jardine and Moore (2016), such that search performance was generally impaired in dynamic displays. Participants were significantly more efficient, however, at searching for the target in the dynamic color condition compared to the orientation condition, $t(26)=4.23, p<.001$. Similarly, the results in the predictability condition suggest that the use of a predictable border improved performance, though the effect was only present with small set size, such that there was an increase in search accuracy relative to that observed in the orientation condition $(t(54)=2.14, p=.037)$. There was a trend toward performance decrements, however, with a larger set size $(t(54)=-2.09, p=.042)$.
Table 4. Descriptive statistics: Mean accuracy (\%) and standard deviation (SD) across conditions in the pilot study

\begin{tabular}{llccc}
\hline Condition & & Mean & SD \\
\hline Orientation conditions & Dynamic & & 75.66 & 22.26 \\
Color conditions & Dynamic circle & & 93.11 & 8.19 \\
Predictability conditions & Set size $=8$ & & 85.69 & 11.44 \\
& Set size $=16$ & & 66.43 & 8.29 \\
\hline
\end{tabular}

\section{References}

Alexander, R. G., Nahvi, R. J., \& Zelinsky, G. J. (2019). Specifying the precision of guiding features for visual search. Journal of Experimental Psychology: Human Perception and Performance, $45,1248-1264$.

Boot, W. R., Kramer, A. F., Becic, E., Wiegmann, D. A., \& Kubose, T. (2006). Detecting transient changes in dynamic displays: The more you look, the less you see. Human Factors, 48, 759-773.

Boot, W. R., Becic, E., \& Kramer, A. F. (2009). Stable individual differences in search strategy?: The effect of task demands and motivational factors on scanning strategy in visual search. Journal of Vision, 9(3):7.1-16.

Braithwaite, J. J., \& Humphreys, G. W. (2003). Inhibition and anticipation in visual search: Evidence from effects of color foreknowledge on preview search. Perception and Psychophysics, 65, 213-237.

Bravo, M. J., \& Nakayama, K. (1992). The role of attention in different visual-search tasks. Perception \& Psychophysics, 51, 465-472.

Carter, R. C. (1982). Visual search with color. Journal of Experimental Psychology: Human Perception and Performance, 8, 127-136. doi: https://doi.org/10.1037/0096-1523.8.1.127

D’Zmura, M. (1991). Color in visual search. Vision Research, 31, 951966. doi: https://doi.org/10.1016/0042-6989(91)90203-H

Duncan, J. (1989). Boundary conditions on parallel processing in human vision. Perception, 18, 457-469.

Duncan, J., \& Humphreys, G. W. (1989). Visual search and stimulus similarity contents. Psychological Review, 96, 433-458.

Egeth, H., \& Dagenbach, D. (1991). Parallel versus serial processing in visual search: Further evidence from subadditive effects of visual quality. Journal of Experimental Psychology: Human Perception and Performance, 17, 551-560.

Green, B. F., \& Anderson, L. K. (1956). Color coding in a visual search task. Journal of Experimental Psychology, 51, 19-24.

Hayakawa, S., Kawai, N., \& Masataka, N. (2011). The influence of color on snake detection in visual search in human children. Scientific Reports, 1(80), 1-4.

Hollingworth, A., \& Henderson, J. M. (2000). Semantic informativeness mediates the detection of changes in natural scenes semantic informativeness mediates the detection of changes in natural scenes. Visual Cognition, 7, 213-235.

Jardine, N. L., \& Moore, C. M. (2016). Losing the trees for the forest in dynamic visual search. Journal of Experimental Psychology: Human Perception and Performance, 42, 617-630.

Nagy, A. L., \& Sanchez, R. R. (1990). Critical color differences determined with a visual search task. Journal of the Optical Society of America A, 7, 1209-1217.

Olivers, C. N. L., Humphreys, G. W., \& Braithwaite, J. J. (2006). The preview search task: Evidence for visual marking. Visual Cognition, 14, 716-735. 
Proverbio, A. M., Burco, F., Del Zotto, M., \& Zani, A. (2004). Blue piglets? Electrophysiological evidence for the primacy of shape over color in object recognition. Cognitive Brain Research, 18, 288-300.

Sireteanu, R., \& Rettenbach, R. (1995). Perceptual learning in visual search: Fast, enduring, but not specific. Vision Research, 35, 2037-2043.

Sireteanu, R., \& Rettenbach, R. (2000). Perceptual learning in visual search generalizes over tasks, locations, and eyes. Vision Research, 40, 2925-2949.

Smallman, H. S., \& Boynton, R. M. (1990). Segregation of basic colors in an information display. Journal of the Optical Society of America A, 7, 1985-1994.

Smilek, D., Enns, J. T., Eastwood, J. D., \& Merikle, P. M. (2006). Relax! Cognitive strategy influences visual search. Visual Cognition, 14, 543-564.

Treisman, A. (1986). Features and objects in visual processing. Scientific American, 255, 114-125. doi: https://doi.org/10.1038/ scientificamerican1186-114B

Treisman, A. M., \& Gelade, G. (1980). A feature-integration theory of attention. Cognitive Psychology, 12, 97-136. doi:https://doi.org/10. 1016/0010-0285(80)90005-5

Wang, Q., Cavanagh, P., \& Green, M. (1994). Familiarity and pop-out in visual search. Perception \& Psychophysics, 56, 495-500. doi: https://doi.org/10.3758/BF03206946

Watson, D. G., \& Humphreys, G. W. (1998). Visual marking of moving objects: A role for top-down feature-based inhibition in selection.
Journal of Experimental Psychology: Human Perception and Performance, 24, 946-962.

Watson, M. R., Brennan, A. A., Kingstone, A., \& Enns, J. T. (2010). Looking versus seeing: Strategies alter eye movements during visual search. Psychonomic Bulletin \& Review, 17, 543-549.

Williams, D. E., Reingold, E. M., Moscovitch, M., \& Behrmann, M. (1997). Patterns of eye movements during parallel and serial visual search tasks. Canadian Journal of Experimental Psychology, 51, 151- 164. doi:https://doi.org/10.1037/1196-1961.51.2.151

Wolfe, J. M. (1998). Visual search. In H. Pashler (Ed.), Attention (pp. 1373). Hove, England: Psychology Press/Erlbaum (UK) Taylor \& Francis.

Wolfe, J. M., Friedman-Hill, S. R., Stewart, M. I., \& O'connell, K. M. (1992). The role of categorization in visual search for orientation. Journal of Experimental Psychology: Human Perception and Performance, 18, 34-49. doi: https://doi.org/10.1037/0096-1523. 18.1.34

Zelinsky, G. J., \& Sheinberg, D. L. (1997). Eye movements during parallel-serial visual search. Journal of Experimental Psychology: Human Perception and Performance, 23, 244-262. doi: https://doi. org/10.1037/0096-1523.23.1.244

Publisher's note Springer Nature remains neutral with regard to jurisdictional claims in published maps and institutional affiliations. 